\title{
Modelling the mechanoreceptor's dynamic behaviour
}

\author{
Zhuoyi Song $^{1 *}$, Robert W Banks², Guy S Bewick ${ }^{3}$ \\ From 24th Annual Computational Neuroscience Meeting: CNS*2015 \\ Prague, Czech Republic. 18-23 July 2015
}

\begin{abstract}
All sensory receptors adapt, i.e., they constantly adjust their sensitivity to external stimuli to match the current natural environment [1]. Electrophysiological responses of sensory receptors from widely different modalities seem to exhibit common features related to adaptation, and these features can be used to examine the underlying sensory transduction mechanisms [1,2]. Among the principal senses, mechanosensation remains the least understood at the cellular level [3]. To gain greater insights into mechanosensory signalling, we investigated if mechanosensation displayed adaptive dynamics that could be explained by similar biophysical mechanisms in other sensory modalities. To do this, we adapted a fly photoreceptor model [4] to describe the primary transduction process for a stretch-sensitive mechanoreceptor, taking into account the viscoelastic properties of the accessory muscle fibres [5] and the biophysical properties of known mechanosensitive channels (MSCs). The model's output is in remarkable agreement with the electrical properties of a primary ending of an isolated decapsulated spindle; ramp-and-hold stretch evokes a characteristic pattern of potential change, consisting of a large dynamic depolarization during the ramp phase and a smaller static depolarization during the hold phase [6]. The initial dynamic component is likely to be caused by both the mechanical properties of the muscle fibres and a refractory state of MSCs. Consistent with literature, the current model predicts that the dynamical component is due to a rapid stress increase during the ramp [7]. More novel predictions from the model are the mechanisms to explain the initial peak in the dynamical component. At the onset of the ramp, all MSCs are sensitive to external stimuli, but as they become refractory (clipped inactivated state), fewer
\end{abstract}

\footnotetext{
* Correspondence: zhuoyi.song@ucl.ac.uk

${ }^{1}$ Centre for Mathematic, Physics and Engineering in the Life Sciences and Experimental Biology (CoMPLEX), University College London, London, UK Full list of author information is available at the end of the article
}

MSCs are able to respond to the continuous stretch, causing a sharp decrease after the peak response. The same mechanism could contribute a faster component in 'sensory habituation' of a mechanoreceptor, in which a receptor responds more strongly to the first stimulus episode during repetitive stimulation [8].

\section{Authors' details}

${ }^{1}$ Centre for Mathematic, Physics and Engineering in the Life Sciences and Experimental Biology (COMPLEX), University College London, London, UK. ${ }^{2}$ School of Biological and Biomedical Sciences, University of Durham, Durham, DH1 3LE, UK. ${ }^{3}$ School of Medical Sciences, Institute of Medical Sciences, University of Aberdeen, Aberdeen, AB25 2ZD, UK.

Published: 18 December 2015

\section{References}

1. De Palo G, Facchetti G, Mazzolini M, Menini A, Torre V, Altafini C: Common dynamical features of sensory adaptation in photoreceptors and olfactory sensory neurons. Scientific Reports 2013, 3:1251.

2. Torre V, Ashmore JF, Lamb TD, Menini A: Transduction and adaptation in sensory receptor cells. J Neurosci 1995, 15(12):7757-7768.

3. Chalfie M: Neurosensory mechanotransduction. Nat Rev Mol Cell Bio 2009, 10:44-52.

4. Song ZY, Postma M, Billings SA, Coca D, Hardie RC, Juusola M: Stochastic, Adaptive Sampling of Information by Microvilli in Fly Photoreceptors. Curr Biol 2012, 22(15):1371-1380.

5. Swerup C, Rydqvist B: A mathematical model of the crustacean stretch receptor neuron. J Neurophysiol 1996, 76(4):2211-2220.

6. Hunt CC, Wilkinson RS, Fukami Y: Ionic basis of the receptor potential in primary endings of mammalian muscle spindles. J Gen Physiol 1978, 71(6):683-698.

7. Matthews PB: Muscle Spindles and Their Motor Control. Physiol Rev 1964, 44(2):219-288.

8. Pasztor VM, Bush BMH: Graded Potentials and Spiking in Single Units of the Oval Organ, a Mechanoreceptor in the Lobster Ventilatory System .3. Sensory Habituation to Repetitive Stimulation. J Exp Biol 1983, 107:465-472.

doi:10.1186/1471-2202-16-S1-P16

Cite this article as: Song et al:: Modelling the mechanoreceptor's dynamic behaviour. BMC Neuroscience 2015 16(Suppl 1):P16. 